\title{
Location Selection of Logistics Center in e-Commerce Network Environments
}

\author{
Baiyu Chen ${ }^{1, *}$, Biying Wang ${ }^{2}$ \\ ${ }^{1}$ College of Engineering, University of California Berkeley, Berkeley, USA \\ ${ }^{2}$ Engineering Institute, Ocean University of China, Qingdao, China
}

Email address:

baiyu@berkeley.edu (Baiyu Chen)

${ }^{*}$ Corresponding author

\section{To cite this article:}

Baiyu Chen, Biying Wang. Location Selection of Logistics Center in e-Commerce Network Environments. American Journal of Neural Networks and Applications. Vol. 3, No. 4, 2017, pp. 40-48. doi: 10.11648/j.ajnna.20170304.11

Received: October 21, 2017; Accepted: November 20, 2017; Published: December 26, 2017

\begin{abstract}
The site selection of logistics center is a very complicated and enormous system problem. Each site selection method and model is based on a certain premise and hypothesis. The site selection decision of distribution center has an important influence on the whole logistics system operation. A good location of urban logistics sites is important for optimizing the logistics network, and improving the urban traffic conditions, and level of logistics services. Motivated by this, in this paper, based on the research of sixteen cities in southeastern China and neural network algorithms, we proposed a logistics center location selection algorithm. Our method considers the six important concepts reflecting the performance index of the city logistics, such as city location quotient, market prosperity degree, proportion of freight volume, urban centricity, per capita gross domestic product (GDP), and population size. Our method conducts the nested fuzzy analytic hierarchy process (AHP), and then investigates the total ranking of the single order hierarchy to select suitable logistics centers.
\end{abstract}

Keywords: Logistics Center Location, Cluster Analysis, Analytic Hierarchy Process, Neural Network, Machine Learning

\section{Introduction}

The location of a logistics center refers to the planning process of selecting one or more addresses and setting up a distribution center in an economic area with a number of supply points and a number of demand points [1]. Better logistics center location scheme can effectively save costs, promote the coordination of production and consumption, and ensure the balanced development of the logistics system. Therefore, the location of the logistics distribution center is reasonable, and will directly affect the delivery efficiency and costs of the entire logistics system. With the rapid development of sensor technology and computer technology today, with the development of modern logistics distribution center towards the "informationized", "digitized" and "networked", the airporter, energy-saving and green one, it's the main direction of development to combine unmanned equipment and logistics industry. Shunfeng express in China, Amazon in USA, United Parcel Service (UPS) and DHL and other well-known logistics enterprises have improved the cross research field of logistics and unmanned. While all kinds of communication network technology mature, network coverage, location based service LBS, Global Positioning System GPS navigation technology and various kinds of sensors and the wireless signal transceiver technology have provided a solid technical support for the development of logistics automation system.

Recently, neural networks [2-4] have witnessed as an emerging area of research because of their widespread applications in image processing, signal processing, combinatorial optimization, associative memories, pattern recognition [5-11]. These applications heavily depend on the dynamic behaviors of neural networks. Inspired by this, in this paper, we divide the study of logistics center location into the following three categories: continuous logistics center location, discrete logistics center location, expert consultation method, logistics center location. The method of continuous logistics center location is to define the optional range of the logistics center in the whole plane, and establish the logistics center at any point on the plane. This method does not have the special limited requirement, therefore, it 
has very good flexibility especially suitable for selecting one or more logistics centers in such an area. However, this kind of method has great limitations due to sometimes the best address obtained by this method being located in the river valley, the mountains, the impossible position [12-15], so when carried out to pick up the location the method has great limitations, not universal. The defect of discrete location of logistics center to overcome the continuous logistics center location is the best place to be implemented. The selection range of the logistics center is a point in the plane, according to a predetermined target to select the best logistics center [16-24]. However, this method establishes the mathematical model for most of Non-deterministic Polynomial NP problem $[1,4]$ which cannot be transformed into a linear model. However, in reality, the use of variables in those methods are often not available. As a result, they are not feasible to solve ours. The former two kinds of quantitative analysis methods of location research in practice is not universal, and ignores the location of many factors, such as: in the study of geological conditions, operating environment, traffic conditions, human development, city space and etc. It is difficult to achieve to constraint these factors into quantity in the model, therefore, in the actual study, using expert consultation method [25-31] of logistics center location more generally, which is the best decision based on numerical form to express expert judgment, after a comprehensive analysis. By this method, the subjective judgment of experts has played an important role, so the inevitable result can be influenced by expert experience, personal preferences, the field of knowledge structure, and many other factors. Obviously, the continuous and discrete location of logistics center logistics center location is more suitable for the selection of distribution center in a region location micro like this, and the expert consultation method of logistics center location method belongs to the qualitative analysis, whose results are lack of certain credibility. Therefore, it is very important to establish a relatively comprehensive evaluation index system of logistics center location.

In this paper, the sixteen cities in southeast coastal China system as the research object of the logistics center location research, proposed can reflect the 6 performance indices of the city logistics: City location quotient, market prosperity, freight volume, the proportion of the city center, the per capita GDP, population, and gives the fuzzy factors [32-36] and fuzzy clustering evaluation based on a more comprehensive nesting level analysis method to select the best place.

\section{Model based on Fuzzy Cluster Analysis}

The location model of regional logistics center can be regarded as a grey system, and the process of location selection includes two stages, macro and micro. At present, the research on the location of macro multi regional city logistics centers is very scarce. In fact, macro site selection is the premise and important reference of microscopic site selection. As a soft division method, fuzzy cluster analysis can comprehensively and accurately classify cities with similar indexes by selecting different indexes and establishing index system. However, after clustering analysis of the city similar to the same, in this way there is no obvious difference between the advantages and disadvantages, therefore, we also need to use hierarchical analysis to get the best logistics center address.

\subsection{Basic Principle of Fuzzy Clustering}

Clustering analysis is based on the analysis of multiple subjects to be classified with the characteristics of the research objects. Through quantitative indicators describing the individual properties, the properties of similar individuals are clustered together that the same class of individual is with a high degree of homogeneity, which is Homogeneous. Those belonging to different categories of individual heterogeneity have high heterogeneity that is heterogeneous. Usually the study sample has many similarities, for multiple observation samples from the same samples group, by finding out some statistic to measure the degree of similarity between the samples or indicators. Classification of these statistics is partition size, the distance function which is defined for each sample in the same index range. By selecting the appropriate size of the standard distance. The distance similar samples (or index) polymerization consist of a class and the samples (or index) with very large distance are divided into different categories. That is clustering analysis.

\subsection{Fuzzy Cluster Analysis Model}

As the object to be classified, each object is represented by $\mathrm{m}$ different indexes, and the value of is expressed as, so that an original data matrix is naturally obtained. Usually, the dimension of the data is unified by the translation to standard deviation transformation and the shift to range transformation [37-41], and the fuzzy matrix in fuzzy clustering is obtained. If the degree of similarity which is the coefficient of similarity [42-48]. The so-called clustering method is based on the fuzzy matrix of the object classification method. Through the selection of confidence level changes, we get different classification results, and then form a dynamic clustering map [49-53]. The commonly used methods are transitive closure method, Boolean matrix method and direct clustering method

\subsection{Selection of Performance Metrics}

In the process of selection of logistics center for multi-area city, this paper presents six performance indexes, and these indexes can reflect the macro factors, reflecting the city logistics properties and at the same time it can quantify: city location quotient, market prosperity degree, the proportion of freight volume, urban centricity, per capita GDP and population size, the six indices can comprehensively measure the logistics level of a city. The specific formula for 
calculating the six indices is given below:

(1) City Location Quotient (CLQ)

City location quotient $=$ (the number of people employed in urban transportation, warehousing and postal service the total number of people employed nationwide)/(the total number of people in the city who are employed the number of people employed in transportation, warehousing and postal service nationwide). Urban transportation, warehousing and postal services are all directly related to the logistics industry, so the city location quotient reflects the degree of specialization of logistics of a city. The larger the quotient, the higher the degree of specialization of urban logistics, and the more suitable is the logistics center to be established in this city.

(2) Urban Centricity (UC)

Urban centricity $=$ (number of urban household telephone total population nationwide)/(number of household telephone nationwide urban population).

The centricity of the city reflects the spatial difference in the degree of unobstructedness of logistics information channel and transmits the degree of convenience of logistics information. The unobstructed flow of information is one of the most basic requirements of the logistics center. The number of household telephones selected in this paper is the number of household mobile phones documented in the statistical yearbook of each city.

(3) Market Prosperity Degree (MPD)

Market prosperity degree is represented by the ratio of the city's sales of wholesale and retail sales to the city's gross domestic product. Market prosperity degree reflects the developmental potential of a regional market and the operating environment of the logistics center.

(4) Proportion of Freight Volume (PFV)

Proportion of freight volume $=$ urban freight volume/nationwide freight volume, which reflects the degree of development of transportation in a region, a good traffic condition is also the basic requirement of logistics center, it is the best for the selected region to realize a combined transportation through sea, railway, land and air.

(5) Per Capita GDP (PCG)

Per capita GDP usually reflects the living standard and consumption level of the people in the region, and indirectly reflects the local market of a place where the logistics center is located.

(6) Population Size (PS)

The population size reflects the size of consumer population in a region, reflecting the number of potential consumers in the city and the needs of the logistics center for the labor force and high quality talents. All of the six indices mentioned above are originally recorded in the statistical yearbook of each city.

\subsection{Model Deriving}

This paper will study the site selection of the logistics center in the optimal cities in the most prosperous southeast coastal region of China. 16 large and medium-sized cities in the southeast coastal areas (suitable for establishing logistics center) are chosen, according to the selected logistics index mentioned above, a clustering analysis on the cities was carried out. Cities that have similar indexes are grouped together, so as to determine the candidate site for urban logistics center.

By referring to data of the sixteen mature cities in 2013 "Statistical Yearbook" in (city transportation, warehousing and postal service employees, the total number of employees, city population, city telephone number of wholesale and retail sales, GDP of the city, city freight, GDP per capita, city population the number, etc.), and in accordance with the above formula to reprocess the data, we get six index data, as shown in Table 1.

Table 1. Comparison of cities based on six indices.

\begin{tabular}{lllllll}
\hline City & CLQ & UC & MPD & PFV & PCG & PS \\
\hline Shanghai & 6.19 & 0.46 & 2.67 & 0.023 & 85373 & 2380.43 \\
Nanjing & 2.14 & 1.72 & 0.39 & 0.01 & 88525 & 816.1 \\
Suzhou & 2.51 & 1.79 & 0.21 & 0.0039 & 113860 & 1054.91 \\
Wuxi & 2.45 & 2.34 & 0.3 & 0.0044 & 165936 & 470.07 \\
Changzhou & 0.82 & 1.88 & 1.23 & 0.0038 & 77485 & 362.86 \\
Zhenjiang & 0.85 & 0.6 & 0.26 & 0.0032 & 83650 & 315.48 \\
Nantong & 2.51 & 0.56 & 0.35 & 0.0065 & 62506 & 729.7 \\
Yangzhou & 0.38 & 1.21 & 0.21 & 0.0027 & 58950 & 460.05 \\
Taizhou & 0.45 & 0.98 & 0.23 & 0.0041 & 58378 & 506.35 \\
Hangzhou & 3.97 & 1.99 & 0.34 & 0.0073 & 88962 & 880.2 \\
Ningbo & 3.64 & 2.29 & 0.32 & 0.0079 & 86228 & 577.71 \\
Huzhou & 5.46 & 1.51 & 0.38 & 0.0047 & 57350 & 261.38 \\
Jiaxing & 2.59 & 1.46 & 0.38 & 0.0044 & 63508 & 454.4 \\
Shaoxing & 6.92 & 1.23 & 0.32 & 0.0023 & 73304 & 494.3 \\
Zhoushan & 9.74 & 1.65 & 0.34 & 0.0049 & 74831 & 114 \\
Taizhou & 17.52 & 1.59 & 0.45 & 0.0052 & 49711 & 600.5 \\
\hline
\end{tabular}

We can see from the above four maps for the six logistics indicators, each indicator corresponding to the maximum value of the city is different. None of the indicators are higher than that of other city, which also reflects the results of cluster analysis that among the types is relatively far distance, there is no good or bad good points. After standardizing process [54-59], the original data by transform standard method, we use Euclidean distance method [60] to construct the standardize the fuzzy similarity matrix. Then, we use the direct clustering method to analyze sixteen cities clustering degree, as shown in Figure 1.

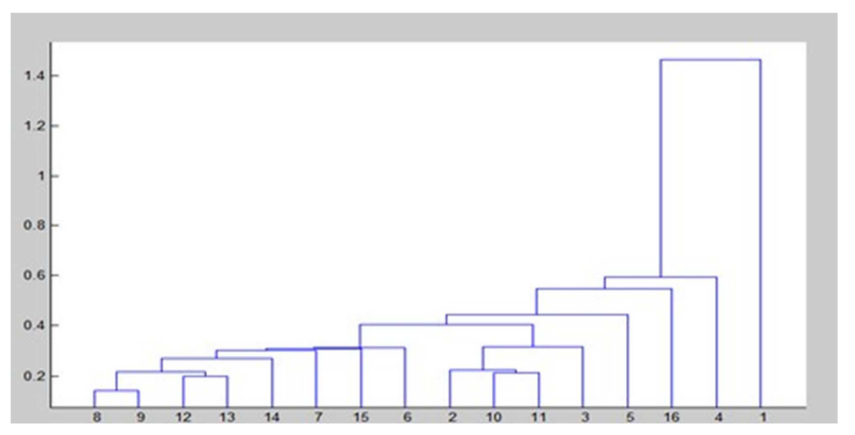

Figure 1. Clustering degree results.

We also see that in every category there are some city logistics index significantly better than the other categories, (all types have different characteristics), and no significant 
difference between various alternatives, so in each class we can choose a city as a logistics center. There is only one city in each class among the first four categories. For the fifth and sixth one after considering the infrastructure factors such as operating environment factors of geographical location, natural environment, and the city in general, the Delphy expert consults that Nanjing and Yangzhou are the most suitable alternative to be the logistics center of the fifth and sixth class. In this way, six logistics centers were selected: Shanghai, Wuxi, Taizhou, Changzhou, Nanjing, Yangzhou.

\section{Optimization}

Of these six logistics center alternatively, using AHP to get the optimal logistics center, and considering the natural environment factors (geographical location, weather conditions, geological conditions, hydrological conditions, terrain conditions, different business environments [61-67], logistics costs, land costs, infrastructure and service level) (traffic conditions, public facilities and other factors (state) government policies, land resources, environmental protection requirements) and other indicators, we can weight coefficient of six city as a logistics center, so as to select the best logistics center.

\subsection{Main Idea of Analytic Hierarchy Process}

Analytic hierarchy process (AHP) is the use of the combination of qualitative and quantitative method to decide some complex and ambiguous problems, it is especially suitable for those problems that hard to be completely quantitatively analyzed, such as the macroscopic problem of site selection of logistics center. It is a multifactor decisionmaking method that is systematic, simple, flexible and practical and has been for the first time invented by the US operational research experts in the early 1970s, being characterized by a combination of quantitative and qualitative methods and being concise, flexible, accurate and scientific, since then, this method has been used to solve the decision-making problems in social, economic and many other fields. When using analytic hierarchy process for modeling, firstly the problem is to be methodized, layered and simplified, namely to construct a hierarchical structure model, and the elements on the higher hierarchy should be used as a principle to dominate the ones on the lower hierarchy, conducting a pairwise comparison between the elements on the lower hierarchy, and then using the relevant measure theory to conduct a pairwise comparison through the importance of element to the higher hierarchical principle, then conducting a scalarization of the judgment by experts with relative scale, and the judgment matrix is established from top to bottom layer by layer, and then the weight of each judgment matrix is solved and the consistency of judgment matrix is checked, finally the weighted comprehensive evaluation of decision-making is obtained and sequenced.

\subsection{Building the AHP Model}

\subsubsection{Hierarchical Structural Model}

The problem in question is divided into three layers: target layer A, principle layer B and program layer C. so the problem has been layered. Concerning the problem of optimal logistics center discussed in this paper, a hierarchical structure shown in the figure 2 can be structured:

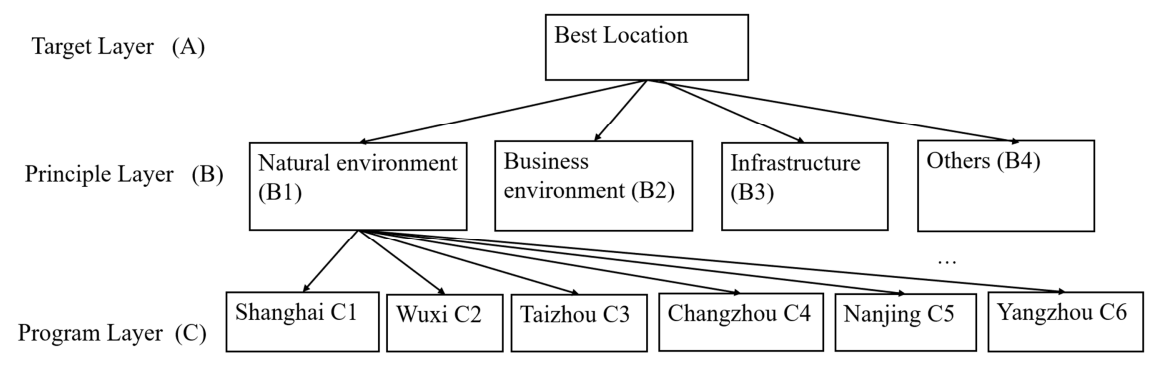

Figure 2. Network of three layers.

\subsubsection{Judgment Matrix}

Every non-bottom element as its membership criteria to compare elements for its importance, now set to compare the influence of $\mathrm{X}=\left\{x_{1}, x_{2}, \cdots x_{n}\right\}$ to a layer of a factor $\mathrm{Z}$ on the level of an element, in order to provide more reliable data, this article takes on every 2 factors and compared to form contrast matrix. That is, the ratio of the sum of the two factors $x_{i}$ and $x_{j}$ whose sum is $a_{i j}$ used to represent each factor's influence. The matrices $A=\left(a_{i j}\right)_{n \times n}$ representing all factor comparison structures which is the pairwise comparison judgment matrix of $\mathrm{X}$ and $\mathrm{Z}$ (referred to as judgment matrix). Obviously if the impact of two factors $x_{i}$ and $x_{j}$ relative to $Z$ is $a_{i j}$, so that $a_{j i}=\frac{1}{a_{i j}}$. According to the above description it can be seen that judging the matrix is the most important mathematical quantity to the analytic hierarchy process. We give some definitions, judgment matrix theorem and properties as the following:

Define: if the matrix satisfies the following two conditions:
a) $a_{i j}>0$
b) $a_{i j}=\frac{1}{a_{j i}}(i, j=1,2 \cdots n)$

Call it positive reciprocal matrix. The values are defined by the numbers $1 \sim 9$ and the inverse as the scale. Table 2 gives the meaning of the importance scale: 
Table 2. Summary of value meanings.

\begin{tabular}{ll}
\hline Value of $\boldsymbol{a}_{\boldsymbol{i} j}$ & Meanings \\
\hline 1 & $x_{i}$ and $x_{j}$ have same important degree \\
3 & $x_{i}$ is slightly more important than $x_{j}$ \\
5 & $x_{i}$ is apparently more important than $x_{j}$ \\
7 & $x_{i}$ is strongly more important than $x_{j}$ \\
9 & $x_{i}$ is extremely more important than $x_{j}$ \\
$2,4,6,8$ & Transition values \\
$1 / 2,1 / 4,1 / 6,1 / 8$ & If $x_{i} / x_{j}=a_{i j}$, then $a_{i j}=\frac{1}{a_{j i}}$ \\
\hline
\end{tabular}

As for the location of logistics center, the business environment is obviously more important than the natural environment, so the comparison value of comparison matrix is 5:1; and the business environment in Shanghai is better than Taizhou, but without a strong dominant. Therefore, the comparison value is $6: 1$.

\subsubsection{Single Hierarchical Arrangement}

The corresponding eigenvectors of the maximal eigenvalue of Judgment matrix A, is identified as the new vector, which is obtained after being normalized is the ranking weight value of importance of the elements on this layer to the ones on the higher layer, this is the process of single hierarchical arrangement. Although this kind of pairwise comparison can reduce the interference of some factors, and objectively reflect the difference in the importance of a pair of factors, it can be inconsistent to some extent in the final comprehensive comparison. But in the last comprehensive comparison, there will be some degree of inconsistency. If the results are completely consistent from beginning to end during the whole comparison (in the most ideal environment), in that way, the matrix A can be identified as a consistent matrix and its elements should also meet the following:

To obtain a scientific and reliable result, the consistency of judgment matrix A should be checked in order to decide whether A is accepted. Furthremore, the consistency of judgment matrix A can be checked by judging whether the equals to $\mathrm{n}$ or not according to the nature of consistent matrix of positive reciprocal matrix A. the greater the is than $n$, the higher the degree of inconsistency of A, as a result, the new vector corresponding to the normalized eigenvector of cannot truthfully reflect the weight of importance of the elements on the lower layer to the ones on the higher layer. Thus, A consistency check is needed to determine the correctness of the judgment matrix. The definition of consistence indicators is:

$$
C I=\frac{\lambda_{\max }-n}{n-1}
$$

Table 3. Consistency degrees.

\begin{tabular}{llllllllllll}
\hline $\mathbf{n}$ & $\mathbf{1}$ & $\mathbf{2}$ & $\mathbf{3}$ & $\mathbf{4}$ & $\mathbf{5}$ & $\mathbf{6}$ & $\mathbf{7}$ & $\mathbf{8}$ & $\mathbf{9}$ & $\mathbf{1 0}$ & $\mathbf{1 1}$ \\
\hline $\mathrm{RI}$ & 0 & 0 & 0.58 & 0.9 & 1.12 & 1.24 & 1.32 & 1.41 & 1.45 & 1.49 & 1.51 \\
\hline
\end{tabular}

The consistency ratio is defined by inquiring the random index table RI (table-3):

$$
C R=\frac{C I}{R I}
$$

If $\mathrm{CR}<0.1$, the judgment matrix is acceptable, otherwise the inconsistency of the judgment matrix is obvious, and the matrix is unacceptable and the judgment matrix needs to be modified.

\subsubsection{General Ranking}

The above all are, the weight vector of elements of the same level relative to some element in the upper layer, that is, the single level sorting. The following is the conclusion that the weight of the plan layer relative to the target is chosen, so that the most appropriate solution is selected.

The following weights are synthesized from top to bottom, and then the hierarchy is sorted, i.e., $\sum_{j=1}^{m} b_{n j} a_{j}$. In other words, the last level (A) contains $m$ factors $A_{1}, A_{2}, \cdots A_{m}$, and their total sort weights are $a_{1}, a_{2}, \cdots a_{m}$. The next layer (B) layer contains $\mathrm{n}$ factors $B_{1}, B_{2}, \cdots B_{n}$, and the hierarchy about $A_{j}$, but the sorting weights are $b_{1 j}, b_{2 j}, \cdots b_{n j}$, so the importance weight of the $\mathrm{B}$ level factors relative to the overall target is:

$$
b_{i}=\sum_{j=1}^{m} b_{i j} a_{j}, i=1, \cdots, n
$$

Similarly, checking the consistency of the total of the model, also from the top to the lower layer, we set the single order consistency index $C I(j) \quad(j=1, \cdots, m)$ obtained by the consistency of judgment of every 2 elements that the corresponding average random consistency index is $R I(j)$, and the consistency ratio of $\mathrm{B}$ layer relative to the overall goal:

$$
C R=\frac{\sum_{j=1}^{m} C I(j) a_{j}}{\sum_{j=1}^{m} R I(j) a_{j}}
$$

$C R<0.1$ shows that the total ranking results of the hierarchy are very good and the analysis results can be correct and acceptable

\subsection{Level Analysis}

By asking the experts to get the comparison matrix of the logistics center with every 2 factors including natural environment, operating environment, infrastructure and other factors, that is, the highest level judgment matrix: 


$$
\mathrm{S}_{0}=\left[\begin{array}{cccc}
1 & 1 / 5 & 1 / 3 & 2 \\
5 & 1 & 2 & 7 \\
3 & 1 / 2 & 1 & 5 \\
1 / 2 & 1 / 7 & 1 / 5 & 1
\end{array}\right]
$$

The location of the logistics center has the greatest impact on the operating environment, followed by infrastructure, and the smallest impact is local policy, environmental protection and other factors. The maximum eigenvalues of positive reciprocal matrices and their corresponding eigenvectors are calculated as:

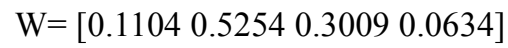

We next conduct consistency checking. First, since the consistency ratio $\mathrm{CR}<0.1$, thus the degree of inconsistency of this judgment matrix is acceptable, and the weight can be replaced by the eigenvector W. Through the expert consultation method, according to comprehensive six city statistical meteorological conditions in 2013 including geological conditions, hydrological conditions, topography and latitude and longitude data and obtain six candidates on the natural environment of the comparison matrix of every 2 as follows:

$$
\mathrm{S}_{1}=\left[\begin{array}{cccccc}
1 & 3 & 1 / 2 & 1 / 3 & 2 & 1 / 5 \\
1 / 3 & 1 & 1 / 4 & 1 / 5 & 1 / 2 & 1 / 7 \\
2 & 4 & 1 & 1 / 2 & 3 & 1 / 3 \\
3 & 5 & 2 & 1 & 4 & 1 / 2 \\
1 / 2 & 2 & 1 / 3 & 1 / 4 & 1 & 1 / 6 \\
5 & 7 & 3 & 2 & 6 & 1
\end{array}\right]
$$

As far as the natural environment is concerned, Yangzhou is the most suitable logistics center, and Wuxi is the least suitable for logistics center. The ratio between Yangzhou and Wuxi is 7:1. Next, we get the comparison matrix of six big cities on the business environment through Delphy expert consultation method and six cities' relevant statistics including land cost, hydropower cost and consumption level and so on in 2013.

$$
S_{2}=\left[\begin{array}{cccccc}
1 & 3 & 6 & 7 & 2 & 8 \\
1 / 3 & 1 & 2 & 3 & 1 / 2 & 4 \\
1 / 6 & 1 / 2 & 1 & 2 & 1 / 3 & 3 \\
1 / 7 & 1 / 3 & 1 / 2 & 1 & 1 / 4 & 2 \\
1 / 2 & 2 & 3 & 4 & 1 & 5 \\
1 / 8 & 1 / 4 & 1 / 3 & 1 / 2 & 1 / 5 & 1
\end{array}\right]
$$

As can be seen from the matrix, Shanghai is the most suitable logistics center in terms of operating environment, and Taizhou has the worst operating environment.

We get the comparison matrix of six big cities on the infrastructure through related data and freight volume data scored by experts in 2013 .

$$
S_{3}=\left[\begin{array}{cccccc}
1 & 5 & 4 & 6 & 2 & 8 \\
1 / 5 & 1 & 1 / 2 & 2 & 1 / 3 & 3 \\
1 / 4 & 2 & 1 & 3 & 1 / 2 & 4 \\
1 / 6 & 1 / 2 & 1 / 3 & 1 & 1 / 4 & 2 \\
1 / 2 & 3 & 2 & 4 & 1 & 6 \\
1 / 8 & 1 / 3 & 1 / 4 & 1 / 2 & 1 / 6 & 1
\end{array}\right]
$$

As can be seen from the matrix, the infrastructure in Shanghai is the best, and the infrastructure in Taizhou is the worst case. Similarly, we get the comparison matrix of six big cities on the other factors according to relevant logistics policies and greening requirements in various cities and experts' scores.

$$
S_{4}=\left[\begin{array}{cccccc}
1 & 1 / 4 & 1 / 7 & 1 / 8 & 1 / 5 & 1 / 9 \\
4 & 1 & 1 / 3 & 1 / 4 & 1 / 2 & 1 / 5 \\
7 & 3 & 1 & 1 / 2 & 3 & 1 / 4 \\
8 & 4 & 2 & 1 & 4 & 1 / 3 \\
5 & 2 & 1 / 3 & 1 / 4 & 1 & 1 / 7 \\
9 & 5 & 4 & 3 & 7 & 1
\end{array}\right]
$$

However, just look at the natural environment factors, Shanghai's natural environment is the worst, Taizhou's natural environment is the best. The same method is used to obtain the ratio of importance weight vectors and consistency tests of the four comparison matrices:

Table 4. Tests on ratios of consistency.

\begin{tabular}{lllll}
\hline & B1 & B2 & B3 & B4 \\
\hline C1 & 0.096 & 0.4245 & 0.4218 & 0.026 \\
C2 & 0.0411 & 0.151 & 0.0934 & 0.0675 \\
C3 & 0.155 & 0.0908 & 0.1451 & 0.157 \\
C4 & 0.2421 & 0.0586 & 0.0601 & 0.2279 \\
C5 & 0.0617 & 0.236 & 0.242 & 0.0839 \\
C6 & 0.4036 & 0.0392 & 0.0377 & 0.4378 \\
CI & 0.0205 & 0.0188 & 0.0198 & 0.0705 \\
CR & 0.0166 & 0.0152 & 0.016 & 0.0568 \\
\hline
\end{tabular}

Table 5. Tests on overall consistency.

\begin{tabular}{lllll}
\hline & B1 & B2 & B3 & B4 \\
\hline & 0.1104 & 0.5254 & 0.3009 & 0.0634 \\
C1 & 0.096 & 0.4245 & 0.4218 & 0.026 \\
C2 & 0.0411 & 0.151 & 0.094 & 0.0675 \\
C3 & 0.155 & 0.0908 & 0.1451 & 0.157 \\
C4 & 0.2421 & 0.0586 & 0.0601 & 0.2279 \\
C5 & 0.0617 & 0.236 & 0.242 & 0.0839 \\
C6 & 0.4036 & 0.0392 & 0.0377 & 0.4378 \\
CI & 0.0205 & 0.0188 & 0.0198 & 0.0705 \\
CR & 0.0166 & 0.0152 & 0.016 & 0.0568 \\
\hline
\end{tabular}

All the CR are less than 0.1 , and the consistency of the four judgment matrices is within the allowable range. The judgment matrix is valid. Through the above analysis, it is easy to get the total weight vector and the total consistency test of the best logistics center location plan layer (third layers) for the overall target (Tier 1). According to the model scheme, C1 establishes the logistics center in Shanghai relative to other regions, as reflected by the weight: 


$$
=0.3622 \text {. }
$$

Thus, the weight vector of the target layer for the target layer is: $(0.3622,0.1163,0.1184,0.09,0.2089,0.1043)$.

Additionally, we have the consistency test as:

$$
\sum_{j=1}^{6} a_{j}(C I)_{j}=0.0226 \sum_{j=1}^{6} a_{j}(R I)_{j}=1.24
$$

We also calculate the total consistency ratio, as: $\mathrm{CR}=0.0226 / 1.24=0.0182<0.1$, which is acceptable.

Table 6. Sorting results for logistics center location selection.

\begin{tabular}{llllll}
\hline City & Shanghai & Wuxi & Taizhou & Changzhou & Nanjing \\
\hline Wt. & 0.3622 & 0.1163 & 0.1184 & 0.09 & 0.2089 \\
\hline
\end{tabular}

We observe from Table 6 that the weight of Shanghai is 0.3622 , which is the biggest weight value, suggesting a most obvious advantage, and thus it is the best places to establish a logistics center. Therefore, if only one logistics center is to be set up in the south China coastal areas (one of the six major economic regions), then it should be set up in Shanghai, if two logistics centers are to be set up, they should be located in Shanghai and Nanjing.

\section{Conclusion}

Concerning the microscopic problem of site selection of multi-area urban logistics center. We present a perspective for consideration that embeds the fuzzy analytic hierarchy process in a more comprehensive manner in this paper. Combining the expert advices, the pairwise judgment matrixes all passed the consistency check, which has verified the rationality of the matrix [81-83], at the same time the pairwise comparison of factors has largely reduced the error brought by the Delphin expert consultation method. The site selection of logistics center is a very complicated and enormous system problem. Each site selection method and model is based on a certain premise and hypothesis. The site selection decision of distribution center has an important influence on the whole logistics system operation. Therefore, only the correct site selection can maximize the effectiveness of the distribution center and meet the demands of consumers, so as to achieve the maximum economic and social benefits.

\section{References}

[1] K. Funahashi, et al. "On the approximate realization of continuous mappings by neural networks." Neural networks 2.3 (1989): 183-192.

[2] E. Badaoui, et al. "The prediction of moisture through the use of neural networks MLP type." Journal of Computer Engineering 11 (2013): 66-74.

[3] W. Abdullah, et al. "Logic mining using neural networks." Proceedings of the international conference on intelligent systems, Kuala Lumpur. 2005.

[4] E. Neda, et al. "Neural network based energy efficiency in wireless sensor networks: A survey." International Journal of Computer Science \& Engineering Survey 1.1 (2010): 39-53.

[5] V. Ponce-López, et al. "ChaLearn LAP 2016: First Round Challenge on First Impressions-Dataset and Results"
Computer Vision-ECCV 2016 Workshops, 2016, pp.400-418.

[6] Z. Chen, et al. "Application of linear mean-square estimation in ocean engineering" China Ocean Engineering, 30(1), 2016, pp. 149-160.

[7] D. Howard, et al. "Neural network design". Martin Hagan, 2014.

[8] H. J. Escalante, et al. "ChaLearn Joint Contest on Multimedia Challenges Beyond Visual Analysis: An overview," 2016 23rd International Conference on Pattern Recognition (ICPR), Cancun, 2016, pp. 67-73. doi: 10.1109/ICPR.2016.7899609

[9] L. Li, J. C. Snyder, I. M. Pelaschier, J. Huang, U.-N. Niranjan, P. Duncan, M., Rupp, K.-R. Müller, K. Burke. "Understanding machine-learned density functionals", Int. J. Quantum Chem. 2016, 116, 819-833. DOI: 10.1002/qua.25040

[10] B. Chen, et al. "Predicting Joint Return Period Under Ocean Extremes Based on a Maximum Entropy Compound Distribution Model", International Journal of Energy and Environmental Science. Vol. 2, No. 6, 2017, pp. 117-126. doi: 10.11648/j.ijees.20170206.11

[11] B Chen, et al. "Cyber-Physical System Enabled Nearby Traffic Flow Modelling for Autonomous Vehicles", 36th IEEE International Performance Computing and Communications Conference, Special Session on Cyber Physical Systems: Security, Computing, and Performance, (IPCCC-CPS 2017), 2017.

[12] S. Pedro, et al. "Improving malware detection accuracy by extracting icon information", IEEE International Conference on Multimedia Information Processing and Retrieval.

[13] F. Brockherde, et al. "Bypassing the Kohn-Sham equations with machine learning", Nature Communications, 8(1):872.

[14] Kevin $\mathrm{Vu}$, et al. "Understanding kernel ridge regression: Common behaviors from simple functions to density functionals", International Journal of Quantum Chemistry, 115(16):1115-1128.

[15] L. Wang, et al. "A new model for calculating the design wave height in typhoon-affected sea areas" Natural Hazards, 67(2): 2013.

[16] X. Xu, et. al., "A New Method to Estimate Wave Height of Specified Return Period" Chinese Journal of Oceanology and Limnology. 2017, 35(5), pp. 1002-1009.

[17] I. Sergey, et al. "Batch normalization: Accelerating deep network training by reducing internal covariate shift." International Conference on Machine Learning. 2015.

[18] Z. Yuke, et al. "Target-driven visual navigation in indoor scenes using deep reinforcement learning." Robotics and Automation (ICRA), 2017 IEEE International Conference on. IEEE, 2017. 
[19] L. Gouveia, et al. "Modeling and simulation of Maximum power point tracker in Ptolemy", Journal of Clean Energy Technologies, Vol. 1, No. 1, 2013, PP 6-9.

[20] Y. Jia, et al. "Caffe: Convolutional architecture for fast feature embedding." Proceedings of the 22nd ACM international conference on Multimedia. ACM, 2014.

[21] I. Sutskever, et al. "Sequence to sequence learning with neural networks." Advances in neural information processing systems. 2014.

[22] W. Cai, et al. "3-Axes MEMS Hall-Effect Sensor," presented by the 2011 IEEE Sensors Applications Symposium, pp 141-144.

[23] M. Long, et al. "Learning transferable features with deep adaptation networks" International Conference on Machine Learning. 2015.

[24] S. Frank, et al. "High Performance SOI RF Switch for Healthcare Application" International Journal of Enhanced Research in Science, Technology \& Engineering, ISSN: 23197463, Vol. 5, Issue 10, 2016, pp 23-28.

[25] W. Cai, et al. "DESIGN OF LOW POWER MEDICAL DEVICE" International Journal of VLSI design \& Communication Systems (VLSICS) Vol.8, No.2, pp1-7, $04 / 2017$.

[26] L. Huang, et al. "2.4GHZ Class AB Power Amplifier for Wireless Medical Sensor Network "International Journal of Enhanced Research in Science, Technology \& Engineering, vol. 5 Issue 4, pp. 94-98 April-2016 ER.

[27] B. Wu, et al. "2.4 GHz Class F Power Amplifier for Healthcare Application" International Journal of Computer Science and Information Technologies, Vol. 7 (3), 2016, pp 1086-1090 (IJCSIT)

[28] P. Zavattieri, et al. "The relationship between cellulose nanocrystal dispersion and strength" Construction and Building Materials, 119, p71-79, 2016.

[29] Y. Cao, et al. "The influence of cellulose nanocrystals on the microstructure of cement paste" Cement and Concrete Composites, 74, p164-173, 2016.

[30] F. Guoliang, et al. "Multi-layer joint gait-pose manifold for human motion modeling," 2013 10th IEEE International Conference and Workshops on Automatic Face and Gesture Recognition (FG).

[31] V. Kevin, et al. "Understanding kernel ridge regression: Common behaviors from simple functions to density functionals" International Journal of Quantum Chemistry 115.16 (2015): 1115-1128.

[32] K. Zhang, et al. "Mining rate optimization considering the stockpiling: A theoretical economics and real option model". Resources Policy, 47, pp.87-94.

[33] X. Tang, et al. "Water Content and Shear Strength Evaluation of Marine Soil after Electro-Osmosis Experiments" Drying Technology, 35(14):1696-1710 2017.

[34] B. Jin, et al. "An adaptive ultrasonic backscattered signal processing technique for accurate object localization based on the Instantaneous Energy Density Level" Journal of Medical Imaging and Health Informatics (JMIHI), vol. 5, no. 5, pp.1059-1064, 2015, American Scientific Publishers, ISSN: 2156-7018
[35] Y. Li, et al. "An integrated location-inventory problem in a closed-loop supply chain with third-party logistics" International Journal of Production Research, published online 2017.

[36] S. Wei et al. "Analysis of wave motion in one-dimensional structures through fast-Fourier-transform-based wavelet finite element method" Journal of Sound and Vibration 400 (2017): 369-386.

[37] X. Cui, et al. "Optimization of a GPU Implementation of Multi-dimensional RF Pulse Design Algorithm," International Conference on Bioinformatics and Biomedical Engineering 2011.

[38] Z. Zhang, et al. "Optimization Design of Coupling Beam Metal Damper in Shear Wall Structures" Applied Sciences 7, no. 22017.

[39] D. Li, et al. "Corrosion Monitoring and Evaluation of Reinforced Concrete Structures Utilizing the Ultrasonic Guided Wave Technique" Journal of Distributed Sensor Networks 10, no. 2 (2014).

[40] H. Shao, et al. "Face recognition based on subset selection via metric learning on manifold. Frontiers of Information Technology \& Electronic Engineering" 16(12), 1046-1058 2015.

[41] D. Park, et al. "A Lookahead Read Cache: Improving Read Performance for Deduplication Backup Storage" J. Comp. Sci. Technol. (2017) 32: 26. January 2017, Volume 32, Issue 1, pp 26-40.

[42] B. Jin, et al. "An adaptive ultrasonic backscattered signal processing technique for instantaneous characteristic frequency detection" Bio-Medical Materials and Engineering (BMME), vol. 24, no. 6, pp. 2761-2770, 2014.

[43] Y. Zaitsev, et al. "Scalable, MEMS-enabled, vibrational tactile actuators for high resolution tactile displays" Journal of Micromechanics and Microengineering, 24(12), 2014.

[44] W. Li, et al. "Learning-based and Data-driven TCP Design for Memory-constrained IoT" Distributed Computing in Sensor Systems (DCOSS), 2016 International Conference on. IEEE, 2016.

[45] Y. Mao, et al. "Laar: Long-range radio assisted ad-hoc routing in manets" Network Protocols (ICNP), 2014 IEEE 22nd International Conference on. IEEE, 2014.

[46] M. C. Chuah, et al. "Scalable Keyword-Based Data Retrievals in Future Content-Centric Networks" Mobile Ad-hoc and Sensor Networks (MSN), 2012 Eighth International Conference on. IEEE, 2012.

[47] H. Harvey, et al. "EDOS: Edge Assisted Offloading System for Mobile Devices" The 26th International Conference on Computer Communications and Networks (ICCCN), 2017.

[48] J. Oak, et al. "DRAPS: Dynamic and Resource-Aware Placement Scheme for Docker Containers in a Heterogeneous Cluster" 36th IEEE International Performance Computing and Communications Conference (IPCCC), 2017.

[49] Y. Mao, et al. "Mobile Message Board: Location-based message dissemination in wireless ad-hoc networks" Computing, Networking and Communications (ICNC), International Conference on. 2016. 
[50] L. Li, et al. "Pure density functional for strong correlation and the thermodynamic limit from machine learning" Physical Review B 94.24 (2016): 245129.

[51] A. Nieto, et al. "The real option value of mining operations using mean-reverting commodity prices. Mineral Economics" 28(1-2), pp.11-22 2015.

[52] S. Escalera, et al. "Overcoming calibration problems in pattern labeling with pairwise ratings: application to personality traits" Computer Vision-ECCV 2016 Workshops, 2016, 419432.

[53] Z. Zhang, et al. "A thermography-based method for fatigue behavior evaluation of coupling beam damper" Fracture and Structural Integrity 40 (2017): 149-161.

[54] L. Wei, et al. "Dynamic Generalization Kanerva Coding in Reinforcement Learning for TCP Congestion Control Design" Proceedings of the 16th Conference on Autonomous Agents and Multi Agent Systems, 2017.

[55] M. Ding, et al. "Multilayer Joint Gait-Pose Manifolds for Human Gait Motion Modeling," in IEEE Transactions on Cybernetics, vol. 45, no. 11, pp. 2413-2424, Nov. 2015.

[56] Y. LeCun, et al. "Deep learning." Nature 521.7553 (2015): 436-444.

[57] Y. Gal, et al. "Dropout as a Bayesian approximation: Representing model uncertainty in deep learning." international conference on machine learning. 2016.

[58] K. Zhang, et al. "An economics strategy for criticality Application to rare earth element Yttrium in new lighting technology and its sustainable availability”, In Renewable and
Sustainable Energy Reviews, Volume 77, 2017, Pages 899915, ISSN 1364-0321

[59] R. Olawoyin, et al. "Index analysis and human health risk model application for evaluating ambient air-heavy metal contamination in Chemical Valley Sarnia". Ecotoxicology and Environmental Safety, 148, pp.72-81.

[60] K. Zhang, et al. "Risk of commodity price, production cost and time to build in resource economics. Environment, Development and Sustainability”, pp.1-24. https://doi.org/10.1007/s10668-017-0003-0

[61] L. Wang, et al. "Risk Analysis and Assessment of Overtopping Concerning Sea Dikes in the Case of Storm Surge", China Ocean Engineering. 28(4), 479-487.

[62] X. Sun, et al. "A maximum-entropy compound distribution model for extreme wave heights of typhoon-affected sea areas", China Ocean Engineering. 26(1): 49-58.

[63] S. Yan, et al. "Multivariate Compound Extreme Value Distribution and Its Application". PERIODICAL OF OCEAN UNIVERSITY OF CHINA, 34(5): 893-902.

[64] D. Liu, et al. "Theory of Multivariate Compound Extreme Value Distribution and Its Application to Extreme Sea State Prediction", Chinese Science Bulletin, 51(23): 2926-2930.

[65] L. Wang, et al. "Application of linear mean-square estimation in ocean engineering", China Ocean Engineering, 30(1), 2016, pp: $149-160$.

[66] L. Wang, et al. "Application of linear mean-square estimation in ocean engineering", China Ocean Engineering, 30(1), 2016, pp.149-160. 